\title{
Conocimiento escolar y conocimiento "disciplinar" del profesor: algunas reflexiones sobre la participación del profesor en la construcción y enseñanza del contenido asociado a las disciplinas escolares
}

\author{
Teacher's School Knowledge and "Discipline" Knowledge-Some \\ Reflections on Teacher's Participation in the Construction and Teaching \\ of Content Related to School Disciplines
}

\author{
Conhecimento escolar e conhecimento "disciplinar" do professor: \\ algumas reflexões sobre a participação do professor na construção e \\ ensino do conteúdo associado às disciplinas escolares
}

Jorge Mario Ortega Iglesias ${ }^{1}$

\section{Resumen}

Algunos de los planteamientos que han suscitado el presente documento parten del hecho de reconocer ciertas diferencias epistemológicas que giran en torno a aquellas características, asumidas como distintivas, respecto del saber que el profesor utiliza para enseñar. En este sentido, se reflexiona en torno a los contenidos de enseñanza con los cuales el profesor trabaja asumiéndose como productos históricos culturales propios, cuyos saberes mantienen una dinámica de reciprocidad intelectual con las disciplinas escolares que conforman el currículo en la escuela. Por otra parte, bajo el amplio espectro de análisis histórico y discusión que guarda la categoría "disciplinas escolares" se reflexiona acerca de la responsabilidad histórica que mantiene el profesor en función de la enseñanza de estas disciplinas, pero sobre todo alrededor de su materialización y dinamización. Con base en este criterio se presume al profesorado como parte activa e importante en la producción del saber disciplinar en el sentido escolar, especialmente de los contenidos con que educa sujetos en el aula de clases y que es pieza clave en la apertura de la comprensión del carácter disciplinar constitutivo del conocimiento profesional docente.

\section{Palabras clave}

Conocimiento escolar, conocimiento disciplinar, disciplinas escolares

\section{Abstract}

Some of the ideas that have led to this document derive from the acknowledgment of epistemological differences that revolve around those characteristics taken as distinctive of the knowledge teachers use to teach. In this sense, this text discusses the teaching contents used by the teacher, taking them as cultural historical products and whose knowledge maintains a dynamic intellectual reciprocity with the school disciplines that shape and define the curriculum at the school. On the other hand, and under the multiple possibilities of historical analysis and discussion that categories such as "School Disciplines" can enable, this text also reflects on the historical responsibility of the teacher regarding the teaching of these disciplines, especially when it comes to their materialization and strengthening. Under this assumption, teachers are believed to be active and important actors in the production of specific knowledge of disciplines at school level, mainly of the contents to educate subjects in the classroom. Hence, the production of school contents has become a key element to understand the fundamental value of this knowledge for teaching as a profession and as a discipline.

Keywords

School knowledge, discipline knowledge, school disciplines

1 Docente de planta de la Universidad del Magdalena. Estudiante del Doctorado Interinstitucional en Educación (DIE), sede Universidad Pedagógica Nacional de Colombia. Correo electrónico: joor212@gmail.com 


\section{Resumo}

Algumas das abordagens que suscitaram o presente documento visam reconhecer certas diferencias epistemológicas em relação com aquelas características, assumidas como distintivas, por parte do saber que o professor utiliza no ensino. Nesse sentido, é possível fazer uma reflexão em torno dos conteúdos de ensino com os que o professor trabalha, pensando-os como produtos históricos culturais próprios, cujos saberes matem uma dinâmica de reciprocidade intelectual com as disciplinas escolares que compõem o currículo na escola. Por outra parte, sob o vasto espectro de análise histórico e discussão que é a categoria disciplinas escolares, é possível fazer uma reflexão em relação com a responsabilidade histórica que matem o professor em função ao ensino dessas disciplinas, especialmente ao redor de sua materialização e dinamização. Sob esse critério, é possível pensar o professorado como parte ativa e importante na produção do saber disciplinar no sentido escolar, especialmente dos conteúdos com os que educa sujeitos na sala de aula e que é chave na apertura da compreensão do caráter construtivo do conhecimento profissional docente.

\section{Palavras chave:}

Conhecimento escolar, conhecimento disciplinar, disciplinas escolares.

\section{Artículo recibido el 29 de enero de 2016 y aprobado el 5 de agosto de 2016}

\section{Introducción}

El reconocimiento social del profesor como intelectual transformador (Giroux, 1997), trabajador de la cultura, es sin duda una apuesta fundamental que pretende, entre otros aspectos, la reivindicación del estatus de la profesión docente. Este posicionamiento ha planteado superar la mirada del profesor como sujeto pasivo operacionalizador de currículo por la de un sujeto activo, que además de participar en la formulación de las propuestas curriculares en el contexto escolar produce saberes propios que forjan y enriquecen la actividad del enseñante. Tal propósito en la aurora de conocimiento producto de la investigación propia del programa internacional de investigación sobre el conocimiento del profesor se ve reflejado en el reconocimiento de la construcción de un tipo de conocimiento particular, que destaca al profesor como un sujeto que representa un papel fundamental en las prácticas de enseñanza, tanto por las estrategias que incorpora durante la enseñanza interactiva para hacer efectivo el aprendizaje en los educandos, como por el tipo de relación que este mantiene con el saber, en especial cuando se habla de producir conocimientos para enseñar (Bolívar, 2005, Carr y Kemmis, 1988; Furió, 1994; García, 1997; Gil-Pérez, 1994; Gimeno y Pérez, 1988; Jackson, 1968; Marcelo, 1993; Martínez, 2000, 2005; Perafán, 2004, 2012, en prensa; Ponte, 2012; Porlán y Rivero, 1998; Shulman, 1986).
El conocimiento del profesor ha sido comprendido de manera privilegiada a partir de la reformulación, transformación o transposición que este hace de la cultura en el contexto escolar, el amplio despliegue de categorías como conocimiento didáctico de contenido (Bolívar 2005; Marcelo, 2002), soporte teórico importante de las didácticas específicas y la lectura tradicional de transposición didáctica (Chevallard, 1991), que aborda la teoría antropológica de lo didáctico, entre otras, constituyen un potente marco teórico que soporta la comprensión del trabajo docente a reconocer-se como un trabajo de reorganización, adaptación, transformación o transposición del conocimiento que ha sido dispuesto para ser enseñado (nos referimos de manera específica a los contenidos constitutivos de las disciplinas escolares). En suma, este es el conocimiento profesional que produce el profesor.

Este hecho, a pesar de que ha constituido un avance importante para comprender las diversas relaciones de los sujetos enseñantes con los objetos de saber propios de la escuela, y de que desempeña un papel determinante en la identificación y el esclarecimiento de un tipo de saber base de la profesión docente que a su vez consolide su profesionalidad, pensamos, no resuelve por completo el problema de comprender el sentido real del conocimiento del profesor como un acto de producción propio. Esta última postura, por el contrario, se centra en una 
comprensión alternativa que reconoce el carácter disciplinar del conocimiento que se le ha atribuido al profesorado y que en realidad es construido necesariamente por este ( $c f$. Perafán y Tinjacá, 2014).

En la perspectiva clásica, de fondo se plantea que el conocimiento del profesor se naturaliza y hace evidente, necesariamente, a partir de procesos de transformación y adaptación, entre otros, de saberes foráneos; es decir, de los saberes disciplinares que históricamente la escuela ha reconocido y aceptado como el capital cultual de la humanidad. Reconocemos que este proceso realmente ocurre en la actividad del enseñante, ya que el sentido disciplinar que adquiere el saber en la escuela está permeado también por un tipo de razón que instituye el cómo en la enseñanza de los contenidos; no obstante, muchas de las tesis que acompañan este argumento plantean por defecto un tipo de reducción sobre las posibilidades de asumir al profesorado como un sujeto pensante que participa de manera significativa en la producción (el sentido) del saber disciplinar inscrito en el contexto escolar, ya no como un saber foráneo al cual necesariamente debe acudir legitimando así la condición de saber dispuesto a ser enseñado sino como una construcción que emerge a partir de los propios actos intencionados que definen, distinguen y caracterizan epistemológicamente la enseñanza de tales saberes, como saberes enseñados.

Por consiguiente, es sugerente ampliar la comprensión del carácter disciplinar del conocimiento del profesor, reconociendo también el aporte y sentido que los maestros producen en los actos de enseñanza, cuya tarea es connatural a todo acto de trabajo y relación intencionada con los objetos de saber (Chevallard, 1991), en particular con los contenidos de enseñanza con los cuales el profesorado ha mantenido una relación histórica, como saber enseñado, $y$ en consecuencia ha posibilitado la emergencia de sujetos aprendices de tales contenidos.

Los desarrollos que a continuación se presentarán, pretenden dar pistas y debatir sobre algunas diferencias respecto de aquellos planteamientos que de manera exclusiva reconocen el saber del profesor como un proceso de adaptación, reorganización y transformación de contenidos, pues "reconocer un conocimiento profesional sin su carácter académico disciplinar no constituye legítimamente un aporte a la reivindicación del profesor como intelectual, como trabajador de la cultura y como productor de conocimiento" (Perafán y Tinjacá, 2014, p. 51).

En consecuencia, pensamos en la identificación, organización, emergencia y distinción del saber disciplinar propio del profesor, el cual también otorga identidad a su conocimiento profesional, más allá de su participación y el tipo de relación que pueda mantener con saberes disciplinares foráneos e incluso con las mismas disciplinas escolares. En este sentido entendemos que gran parte de los saberes escolares con que el profesor educa y forma individuos son el resultado de un proceso de producción histórico-cultural con el cual el profesor ha devenido sujeto productor y enseñante del contenido asociado a las disciplinas escolares.

Algunos aspectos históricos que han definido un tipo de comprensión sobre la participación del profesor en la producción de conocimiento asociado a las disciplinas específicas que circulan en el contexto escolar

A mediados de la década de los ochenta los trabajos de Elbaz (1981) sobre el conocimiento práctico del profesor constituyeron una crítica importante respecto de la participación casi nula del profesorado en la configuración de las propuestas curriculares y a su vez en la enseñanza de los contenidos mismos, evidenciando con ello el papel pasivo de los maestros guiados por un tipo de racionalidad técnico-instrumental que definía su aporte y participación en el currículo escolar. Con la noción de conocimiento práctico, que pretendía la identificación de un cuerpo de conocimientos propio del profesorado, producto de su práctica misma, se intentaba cambiar hacia una dinámica activa que visibilizara el aporte de los maestros, en la medida en que fuesen reconocidas las características y el aporte del saber derivado de la práctica profesional, además de las atribuciones de sentido personal que los profesores hacían en la enseñanza y el conjunto de interacciones en el medio escolar de las cuales emerge el saber del profesor en el contexto educativo. 
Este tipo de propuestas, conjuntamente con los trabajos de Schön (1998) sobre la reflexión en la práctica, y de Conelly y Clandinin (1984) en torno al conocimiento práctico visto como filosofías personales, ritos, imágenes, unidades narrativas y ritmos, intentaban en su momento reivindicar el rol del maestro en este escenario. Sin embargo, entender la producción de conocimiento del profesor como aporte válido en el marco de la enseñanza de las disciplinas en la escuela generó menor alcance, por tratarse de un saber de orden práctico que solo daría respuesta a situaciones específicas e inmediatas de la práctica; se basaba en descripciones de situaciones muy personales, "alejado en gran medida" del contenido de la enseñanza. Por supuesto, un lugar poco equiparable a la luz del reinante saber disciplinar que mantendría su lugar preponderante en la escuela.

El aporte en este sentido del conocimiento práctico y los trabajos inspirados en la reflexión producto de la práctica profesional, a pesar de mantener serias críticas y diferencias en comparación con el significado y valor del saber disciplinar que habitaba en la escuela, abrió una gran puerta para entender que tal tipo de saberes son constitutivos de un tipo de epistemología propia, que responden por naturaleza a unos intereses y necesidades del mundo de la práctica, en este caso a procesos de enseñanza propios de la comunidad de profesores.

Seguido de esto, y en medio de una de las más importantes reformas educativas de las últimas décadas en los Estados Unidos, emerge la categoría "conocimiento pedagógico de contenido" (PCK), considerada como las formas o modos en que los profesores comprenden y representan el contenido a sus estudiantes (Shulman, 1986, 1987). Esta potente categoría se mostraba como una propuesta innovadora por tratar de reconciliar, entre otros, uno de los problemas más visibles relacionados con la formación del profesorado: la unión entre el componente de formación disciplinar y el componente de formación pedagógica, es decir el contenido y el cómo enseñar el contenido. Así, Shulman, a partir de la introducción del PCK y su modelo de razonamiento y acción pedagógica abrió un interesante campo de estudios que durante la década de los noventa tuvo un fuerte auge e interés por parte de las comunidades de investigadores a nivel internacional, preocupadas por este tipo de fenómenos inscritos en la enseñanza.

Este importante hecho no solo representó la apertura de un programa de investigación que ha dado cuenta de manera fructífera de la estructura y dinámica del conocimiento del profesor, sino también, y en el marco de la perspectiva de análisis que abordamos sobre el profesor como productor de conocimiento -saber disciplinar escolar-, significó una pauta a seguir.

Si bien el PCK nace en la perspectiva de reconocer un saber base para la enseñanza (Wilson, Shulman y Rickert, 1987), unir el peso que traen consigo la tradición, el rigor y la legitimidad del saber disciplinar junto a la organización del conocimiento pedagógico que ocupa su lugar en el ámbito escolar representaría una compleja labor para comprender en términos teóricos y mucho más en términos prácticos esta amalgama a la cual el profesor debe recurrir para enseñar los contenidos del currículo. La principal respuesta a ello fue el modelo de razonamiento y acción pedagógica, cuya naturaleza intenta "describir cómo los profesores comprenden la materia y la transforman didácticamente en algo 'enseñable' [...] es clave en este proceso el paso del conocimiento de la materia [...] al CDC [o PCK]" (Bolívar, 2005, p. 6). Bajo este principio, entendemos que el acto de razonar pedagógicamente los contenidos de enseñanza en el profesor supone realizar transformaciones del conocimiento de la materia en contenidos que se pueden enseñar y que los estudiantes pueden comprender fácilmente.

La relación del profesor con los contenidos escolares ahora estaría guiada por el principio transformador del contenido disciplinar en contenidos enseñables cuyo producto principal es el PCK. En suma, el conocimiento del profesor como conocimiento pedagógico de contenido obedece fundamentalmente al acto de transformación del saber disciplinar, cuyo saber en últimas corresponde con el saber enseñado. El PCK desde esta perspectiva 
fue la apertura a una avalancha de nociones y categorías que otorgan privilegio a procesos relacionados con el cómo; es decir, la preocupación principal se centró en comprender el conocimiento del profesor en función del cómo enseñar, de las estrategias que utiliza, lo que en la literatura se evidencia como los tipos de representación que emplea el profesor sobre un saber específico en su enseñanza. Esta lectura inclinó la mirada hacia un tipo de comprensión sobre el carácter disciplinar del conocimiento del profesor, cuyo lugar muchas veces es externo a los propios actos que por naturaleza obedecen al mundo escolar, los cuales son necesariamente contextualizados y principalmente obedecen a sujetos históricos que trabajan de forma intencionada con el conocimiento.

Tal hecho ha consolidado un tipo de plataforma teórica que identifica el carácter disciplinar del conocimiento del profesor, de manera exclusiva, desde los lugares de las disciplinas científicas, lo cual, al parecer, desconoce y relega los alcances y características epistemológicas posibles del saber que produce el profesorado responsable de la enseñanza de las disciplinas escolares, y a su vez del continente de contenidos constitutivos de las mismas. Así pues, más allá de encontrar el sentido disciplinar constitutivo de la profesionalidad de la profesión docente en un saber foráneo de referencia, sería importante encontrar desde el contexto del aula, desde la misma enseñanza interactiva, el sentido que este imprime en sus actos de enseñanza como construcción localizada y propia, inclusive, más allá de la perspectiva que lo asocia a actos de adaptación o modelación del saber.

Otro de los aspectos clave-que reconocemos ha dado un fuerte impulso al ámbito del conocimiento profesional del profesor, en especial en la comprensión de su carácter disciplinar- ha sido un tipo de lectura clásica sobre los trabajos del francés Yves Chevallard (1991) a través de su polémica, discutida y estudiada obra La transposición didáctica. Del saber sabio al saber enseñado. Este autor desarrolló un marco teórico potente que ha permitido, entre otros asuntos, el esclarecimiento sobre la distinción epistemológica entre didáctica y didácticas específicas, y con ello, novedosas propuestas orientadas a la enseñanza de los contenidos escolares.
Hacemos especial énfasis en la palabra polémica, ya que Chevallard en su texto deja entrever la riqueza y variedad de interpretaciones a la que tales desarrollos dieron lugar.

Nadie parece haber formulado verdaderamente el problema epistemológico que planteó, junto con algunos otros el concepto de transposición didáctica. ¿Se tratará simplemente de un concepto migrador -quizás un poco más que los otros-, hábil para recorrer en un nomadismo más o menos controlado, el traje de Arlequino del campo de las didácticas, cualificando obstinadamente una forma invariante acoplada a sustancias cambiantes? ¿O acaso habrá encontrado, más allá del desmembramiento ferozmente afirmado de un campo plural, su lugar natural, su espacio vital, desde el cual puede desplazarse fácilmente para adoptar una forma concreta aquí y allá? (Chevallard, 1991, p. 141) [Énfasis del autor].

Una de las más interesantes en la discusión que nos ocupa -insistimos, no la única- es la del saber enseñado, cuya naturaleza en síntesis responde al objeto de estudio de las didácticas específicas. El saber enseñado, bajo el lente de las didácticas específicas, responde a los actos transpositivos del saber, donde el profesor cuenta con un alto grado de responsabilidad para su contribución.

El termino transposición didáctica desarrollado por Chevallard (1985), designa un proceso fundamental, constitutivo de todo dispositivo escolar de enseñanza, que permite el paso de un contenido, de un determinado saber a una versión didáctica de ese objeto. El papel del profesor consiste en didactizar los saberes, recontextualizarlos para transformarlos en objetos de enseñanza, ponerlos en circulación en la clase y asegurar las transformaciones necesarias para facilitar su adquisición (Dolz, 2011, pp. 99-100) [Énfasis del autor].

El saber enseñado se dinamiza en este sentido a través de la transposición didáctica, la cual representa, entre otros actos transpositivos ${ }^{2}$, la transformación que el profesor realiza del saber sabio (académico, bajo una perspectiva clásica del término) a formas

2 Chevallard (1991) también reconoce transposiciones didácticas a nivel institucional. 
susceptibles de ser enseñadas (didactizadas) y fácilmente comprendidas por los educandos. Se plantea con ello su participación en la enseñanza de una disciplina escolar específica.

En este sentido, el trabajo del profesor con los objetos de enseñanza y su contribución a la aparición de los mismos pareciese ser vaciado de sentido, de significado, es delimitado, formado y circunscrito a un solo lugar, por un lente particular (las didácticas específicas), quizás expuesto a un solo tipo de comprensión que supone de manera privilegiada procesos de modelación, transformación, recontextualización. Es decir, su trabajo es "didactizar" los saberes que provienen de un campo disciplinar específico, el cual es responsable de enseñar. Tal aporte significativo que posesiona y consolida la profesión docente no se pone en duda; la pregunta circula más en función del sentido que construye el profesorado como unidad subjetiva cargada de significado histórico, que ha posibilitado de manera particular la enseñanza de contenidos escolares en función de educar sujetos.

Insistimos, no se trata de reivindicar el carácter disciplinar del conocimiento del profesor de manera exclusiva bajo la aurora de un saber disciplinar específico de referencia o de condición externa, que en última instancia ya ha sido reconocido en el ámbito profesional, mucho antes de que el profesorado ingresara a la escuela. Por el contrario, la intención se centra en el posicionamiento del conjunto de atribuciones y construcciones de significado que realizan los profesores cuando enseñan las nociones y los conceptos pertenecientes a las disciplinas que circulan en el entorno escolar. Ello sería una consideración para la apertura del carácter disciplinar propio de la profesión docente, que soporta al mismo tiempo características epistemológicas propias del conocimiento profesional del profesor.

Asimismo, en el plano de las didácticas específicas, el trabajo académico y cultural desarrollado por diversas comunidades de didactas en función de la consolidación y legitimación científica de estos cuerpos disciplinares emergentes ha afianzado aún más esta lectura y comprensión del trabajo docente, asociado a didactizar los saberes escolares de referencia como se ha planteado, en el que la categoría conocimiento didáctico del contenido (CDC) (Bolívar, 2005; Marcelo, 2002) ha constituido también un suelo epistemológico común.

En efecto, el $\mathrm{CDC}^{3}$ deudor del PCK ha representado para la investigación didáctica en la formación del profesorado una de las contribuciones más poderosas (Marcelo, 1993). Dicha categoría, junto al gran impulso teórico aportado por la transposición didáctica y la teoría antropológica de lo didáctico (Chevallard, 1991) se han convertido en piso epistemológico y, a su vez, en una forma de legitimación científica de las didácticas específicas. Esto ha provocado no solo un importante espectro de conocimientos en función de la especificidad que encierra la enseñanza de los contenidos asociados a las disciplinas escolares (con mayor desarrollo en las matemáticas y las ciencias) (Bolívar, 2008) sino que, adicionalmente, tales "didácticas específicas han dado pasos gigantescos para alcanzar su autonomía y madurez disciplinar y han logrado demostrar un nivel de autoorganización francamente envidiable desde el punto de vista del profesorado" (Perafán y Tinjacá, 2014, p. 54). Resolviendo con ello aparentemente el cúmulo de problemas que históricamente han presentado la enseñanza de las disciplinas científicas en la escuela, en especial en función de la representación, muchas veces, distorsionada y desfigurada, que los profesores hacen de tales saberes.

De acuerdo con esto, la solidez disciplinar que demuestran las didácticas específicas es posible gracias al trabajo investigativo que las comunidades de didactas adelantan (Camilloni, 2007). Es un trabajo serio y especializado que tiene por objeto estudiar los fenómenos de enseñanza y aprendizaje propios de las materias escolares, las relaciones complejas constitutivas del sistema didáctico y la transmisión y la apropiación del saber en un contexto escolar (Dolz, 2011), en cuanto, como producto concreto del trabajo de estas comunidades, se generen pautas que

3 Con un potente despliegue en el contexto español, reconocida inicialmente como una traducción del Pск (Marcelo, 1993). 
garanticen una adecuada enseñanza de las disciplinas escolares para la escuela en general y de los contenidos específicos por parte del profesorado en particular.

No obstante, es claro que bajo estos argumentos se presupone que el papel del profesor y la comprensión de su conocimiento profesional, a la luz de la dimensión personal y práctica, se relaciona más con el dominio y desarrollo que del conocimiento didáctico disciplinar, propio de los especialistas, pueda alcanzar el profesorado (Perafán, 2012). Así, en este lugar de interpretación, el trabajo y una responsabilidad crucial del profesor en la enseñanza, como ya lo hemos planteado, consiste en didactizar los saberes académicos de referencia para su formalización en objetos de enseñanza, como también garantizar las posibles transformaciones que faciliten su circulación en el aula y adquisición por parte del estudiantado (Dolz, 2011).

En términos de Perafán y Tinjacá:

...para la tradición latina el CDC es un asunto de especialistas, de investigadores en didácticas específicas que pueden dar cuenta, por una parte, de la manera como la ciencia didáctica produce sus propias entidades conceptuales; por otra parte, de la manera como el profesor apropia tales entidades o conocimientos disciplinares (insistimos: los construidos en el campo de la didáctica con autonomía epistémica); en tercera instancia, de los saberes acerca de cómo enseñar esos conocimientos; por último, de la manera como dichos saberes se integran. En esta tradición el conocimiento profesional docente, entendido desde la dimensión personal y práctica, está asociado mayoritariamente (de manera a veces tácita, otras explícita), a la apropiación y desarrollo que del conocimiento didáctico disciplinar, producido por los especialistas, logre hacer el profesorado. Desde el punto de vista del profesor esto puede ser leído como un desplazamiento de tutor; no necesariamente como la conquista de un campo propio. (2014, p. 55)

Así, el lugar hegemónico que ocupaban las disciplinas científicas en el contexto escolar pasaría a ser ocupado por la comunidad de especialistas encargados de garantizar una adecuada enseñanza de tales disciplinas. Lo anterior no pone en duda la riqueza y los aportes que las didácticas específicas despliegan y que efectivamente generan pautas para mejorar el proceso de enseñanza y aprendizaje, la inquietud está más hacia el posicionamiento del sentido que los maestros, desde el ámbito profesional, construyen de los contenidos de enseñanza, los cuales permiten la emergencia de sujetos aprendices de tales contenidos y que en definitiva pareciese encontrarse aún oculto.

Como consecuencia, nos es preciso preguntar de nuevo por el papel y la participación del profesor en la construcción y materialización de las disciplinas escolares, en particular de los contenidos que enseña, cuyo aporte, como se ha intentado mostrar hasta ahora, pareciese que solo obedece a procesos de transformación de un saber de referencia, al que necesariamente por un impulso casi que involuntario se rinde homenaje por su peso y tradición en la escuela. Este hecho desconoce el sentido y la naturaleza epistemológica característica de la emergencia del conocimiento que produce y con que trabaja el profesor, inscritos en una cultura escolar y dinámica del aula propia, más allá de procesos de modelación de saberes foráneos.

En términos generales, luego de hacer un rápido análisis de estos aspectos históricos que han posicionado un tipo de comprensión sobre el carácter disciplinar del conocimiento del profesorado, no cabe duda de que la profesionalidad docente se acentúa a partir del trabajo que los maestros realizan con los objetos de enseñanza, principalmente en función de procesos de adaptación, transformación y recontextualización de un saber de referencia (disciplinar o dispuesto a ser enseñando), cuyo trabajo representa el aporte que este hace al contenido de las disciplinas escolares que enseña desde el aula de clases. Planteamientos como estos, a nuestro juicio, sitúan en un lugar de menor privilegio la mirada del profesor como intelectual que aporta a partir de saberes propios, producto de su devenir histórico cultural como docente, en la construcción y enseñanza de los contenidos asociados a las disciplinas escolares.

En adelante nos ocuparemos, por lo tanto, en discutir algunos de los siguientes interrogantes, que consideramos determinantes para intentar mostrar otra perspectiva en función de la visibilización del 
saber propio del profesor y la apertura en la comprensión de su carácter disciplinar constitutivo de su profesionalidad: ¿qué se entiende por las disciplinas escolares? ¿Cómo se asume la producción y materialización por parte del profesor de las disciplinas escolares? ¿Cómo se asume bajo esta perspectiva de análisis el conocimiento del profesor?

\section{Disciplinas escolares y conocimiento del profesor}

Cuando usamos el término disciplina, necesariamente nos ubicamos en el plano epistemológico que apunta a un nivel de reflexión sobre la estructuración de los saberes (Gómez y Alzate, 2010). En este caso, indudablemente, la escuela se vislumbra como la institución cultural que encuadra y define el tipo de saber que nos ocupa. Queremos hacer precisiones sobre algunos planteamientos diferenciadores entre disciplina científica y lo que tradicionalmente conocemos como "materia escolar" (Chervel, 1991; Goodson, 1991, 1995; Tanner y Tanner, 1990), los cuales señalan cierto grado de autonomía e independencia que las distinguen e instalan bajo propósitos diferentes.

Las disciplinas escolares, desde una perspectiva social, crítica e histórica (Chervel, 1991; Cuesta, 1997, Goodson, 1991, 1995; Julia, 2000; Popkewitz, 2010; Viñao; 2012; 2014), reconocen un lugar característico e importante al saber que circula en la escuela y que constituye, entre otros aspectos, una forma naturalizada del currículo escolar. Estos saberes de orden disciplinar, como productos históricos de la cultura escolar, operan según Viñao (2012) como organismos vivos;

... nacen, evolucionan, se transforman, desaparecen, se fagocitan y engullen unas a otras, se desgajan, compiten, intercambian información, se aíslan, emparejan o forman tríos e incluso comunas, se atraen, se repelen o son indiferentes entre sí, tienen un nombre que las identifica, cambian de denominación y de apariencia, se jerarquizan, se hacen préstamos, se roban entre ellas, marcan su territorio. ( p. 115)

Validando de manera especial una dinámica compleja que solo cobra sentido al estudiarla desde el contexto escolar y en las prácticas de enseñanza en particular. En este orden, entendemos que tal saber representa una creación histórica de la propia escuela, por y para la escuela (Chervel, 1991), hecho que supera a aquellas posturas que consideran el saber escolar como un saber "banalizado", "vulgarizado" y además "deformado" de las disciplinas científicas. El sentido de las disciplinas escolares tiene otro lugar de anclaje y comprensión, incorpora una condición de independencia en relación con cualquier otro tipo de saber, señala su lugar de origen y desarrollo en la propia dinámica escolar. La disciplina escolar comprendida de esta forma dejaría de ser una simple proyección al margen del saber sabio e iniciaría precisamente cuando se crea lo enseñable; su lugar se ubicaría, por lo menos, en frente de este último, bajo una relación de "autonomía relativa" (Chiss, 1989, citado en Gómez y Alzate, 2010).

Sin embargo, una perspectiva de análisis, en la cual consideramos necesario profundizar, tiene que ver con la enseñanza de los contenidos que constituyen a estas disciplinas, cuya organización y actividad funcional en el aula se debe en gran parte a la participación que tiene el profesor para su producción y enseñanza, es decir, su materialización en el aula de clases, como fenómeno educativo. Para Viñao (2012), un aspecto fundamental en la historia de las disciplinas, en su proceso de formación y cambio a lo largo del tiempo de los códigos disciplinares, es precisamente el elemento humano, en este caso los profesores, quienes en su trabajo de enseñanza las justifican, problematizan su valor formativo y declaran su existencia como tal disciplina académica.

Popkewitz (2004), en un interesante análisis sobre estudios de las matemáticas en el currículo, introduce a manera de metáfora la noción de alquimia para llamar la atención respecto de las "herramientas" de transporte y traducción del currículo escolar. Al igual que la alquimia, la pedagogía es una práctica que de forma mágica transforma las ciencias, la ciencia social y las humanidades en las asignaturas escolares (Popkewitz, 2004). La alquimia $\mathrm{y}$ en especial los alquimistas en el siglo XVII intentaban convertir los metales comunes en oro puro, elemento que socialmente representaba una fuente incomparable de riqueza. 
Este autor plantea que la escuela requiere de prácticas como la alquimia, ya que su papel principal en relación con la enseñanza de los contenidos de las disciplinas escolares está orientado a la formación de niños y niñas, de manera distinta de un sujeto formado para la física o matemática (disciplinas científicas) que responde a intereses y un campo de acción específico. La alquimia, en este sentido, se entiende como actos de creación escolar y no copias de las disciplinas o formas análogas de transformación del saber académico. Así, el trabajo del alquimista representa en nuestros tiempos el trabajo del profesor, que, en el marco de la enseñanza y su trabajo con las disciplinas escolares (a través de procesos alquímicos), participa en gran medida de la creación de los contenidos de estas disciplinas (oro, fuente incomparable de riqueza) con que son educados los sujetos.

Popkewitz (2010) también encuadra estos procesos alquímicos en estructuras participativas que otorgan identidad a las materias escolares, cuya organización da cuenta de tesis culturales, inscritas en un contexto, provenientes de colectivos y actores escolares, alrededor de quién es el niño y qué debe ser.

Estas tesis culturales, que además de actuar como formas de participación con las cuales los profesores enseñan los contenidos del currículo, son también creaciones humanas de sujetos históricos (el profesorado) que inscritos en la cultura escolar actúan no solo como agentes transformadores o modeladores del saber, sino como sujetos activos, visibles y productores de sentido respecto de qué se enseña y para qué. Es a su vez un conocimiento de carácter histórico que se genera en el seno de comunidades escolares, hablamos en especial de comunidades de profesores preocupados por la formación de los sujetos desde el saber específico que les ocupa (matemáticas, física, química, tecnología, etc.). Este hecho atribuye al profesorado una dinámica de reciprocidad intelectual con las disciplinas escolares; es decir, no solo tienen la responsabilidad histórica de enseñar el capital cultural escolar, que en esta vía enriquece su saber profesional y académico, sino en el marco de la relación histórica que han guardado con el saber (contenido disciplinar escolar), bajo el acto intencionado que tienen de enseñarlo, producen conocimiento que otorga a su vez identidad a estas disciplinas.
Ahora bien, de manera particular en la línea de estudios del conocimiento del profesor, Perafán (2013, en prensa), haciendo una lectura alternativa sobre la noción de transposición didáctica, plantea tres tesis que identifican de forma distinta el saber académico del profesor, componente constitutivo del sistema de ideas integrado que otorga identidad a la categoría "conocimiento profesional específico del profesorado"4. Estas tesis también dan cuenta de la relación dinámica y compleja en el marco de la producción y enseñanza de los contenidos escolares: la primera tesis ubica la transposición didáctica ${ }^{5}$ como un fenómeno antropológico y epistemológico, que debe entenderse como el estatuto epistemológico fundante del saber académico específico (contenidos, conceptos u otras nociones empleadas en la enseñanza) del profesor. Tal consideración no solo desplaza a la disciplina científica como saber fundante, sino que revela un falso problema didáctico asociado a la imagen de los saberes disciplinares en el campo escolar y que en este caso ha definido el carácter disciplinar del conocimiento del profesor.

La segunda tesis plantea la comprensión del saber académico del profesor a partir de obstáculos epistemológicos en el sentido bachelardiano. Los saberes académicos son asumidos como objetos de deseo; es decir, el profesor además de ser un sujeto intencional, es un sujeto pulsional que produce conocimiento a partir de la integración de estas características subjetivas. Y la última tesis da cuenta de una solicitud para tratar de superar el miedo al parricidio en la conciencia del profesorado. Esta solicitud se convierte además en una propuesta para desplazar una falsa identidad profesional del cuerpo docente, al reconocerse más en el lugar de las disciplinas científicas que en el contexto escolar propio del trabajo del enseñante.

4 El conocimiento específico del profesorado, según Perafán (2011), es un sistema de ideas que integra saberes académicos, saberes basados en la experiencia, teorías implícitas y guiones y rutinas. Esta integración a manera de fenómeno polifónico de producción de sentido provoca las categorías de enseñanza y contenidos específicos de las disciplina escolares con las que educan los maestros.

5 Plantea que dicha transposición, lejos de ser una estrategia de modelación, constituye un estatuto epistemológico fundante que da cuenta de una forma particular de producción de conocimiento y que es distinta del conocimiento cotidiano o conocimiento científico. 
En este plano, el conocimiento del profesor, más allá de situarse bajo posturas que lo asocian con versiones deformadas del saber disciplinar o formas didactizadas de un saber de referencia (validadas por una comunidad académica externa), representa para las disciplinas escolares un aporte sustancial, en la medida que sea reconocido su estatus y las formas en que, desde el aula de clases, los profesores contribuyen al crecimiento y la consolidación de las mismas.

En esta perspectiva, se intenta precisar un poco sobre el carácter disciplinar constitutivo del conocimiento profesional del profesor, el cual no solo se define a partir de saberes disciplinares foráneos que, lógicamente, han posibilitado la formación de los maestros, sino que en el marco de los actos intencionados que concurren durante la enseñanza interactiva, ya sean denominados como "procesos alquímicos" o comprendidos a través de la lectura alternativa de "transposición didáctica" planteada por Perafán, los contenidos asociados a las disciplinas escolares adquieren por defecto una característica epistemológica distintiva, no precisamente en el plano del saber sabio (disciplinas científicas), o en el mejor de los casos en el lugar del saber dispuesto a ser enseñado (entendido como las disciplinas escolares trabajadas por expertos), sino como el resultado del saber enseñado atribuido a una especie de co-nacimiento, producto, entre otros aspectos, de la subjetividad profesoral cargada de historia e intención propia de enseñar, esta última distintiva y fundante de la subjetividad mencionada.

\section{Conocimiento profesional específico del profesor: un referente de construcción del saber disciplinar escolar del profesorado}

El lugar de la reflexión que este apartado ocupa tiene que ver con la multiplicidad de mundos y voces partícipes en el aula de clases, cuya organización y dinámica propia da cuenta, además de otros aspectos, de la configuración de los contenidos de enseñanza que hace el profesor. En este sentido incorporamos al análisis la categoría conocimiento profesional específico, que a nuestro juicio pretende ser una respuesta coherente respecto de la participación del profesorado en la construcción de los contenidos de enseñanza y el saber disciplinar instituyente de su accionar.

En un acercamiento un poco desde la relación profesor y contenidos escolares durante las prácticas de enseñanza, la apuesta discursiva que aflora en este escenario como lugar de encuentro y creación compleja de sentido define una perspectiva de estudio que intenta comprender en cierta medida cómo el profesorado construye conocimiento desde sus prácticas de enseñanza. La categoría conocimiento profesional específico del profesor asociado a categorías particulares, planteada por Perafán (2015, en prensa), puede representar una vía interesante de análisis para estudiar este fenómeno.

El conocimiento profesional docente específico, asociado a la construcción del sentido de las categorías o nociones concretas que enseña el profesor, según Perafán (en prensa), se define como un sistema de ideas integrado, con cuatro estatutos epistemológicos fundantes asociados (lugares de producción de sentido) que dan cuenta de forma particular de la emergencia de conceptos o nociones con los cuales trabaja el profesor responsable de la enseñanza de las disciplinas escolares. Los sistemas de ideas que integran este tipo de conocimiento son: los saberes académicos del profesor, los cuales ya hemos abordado de manera rápida en páginas anteriores y son asumidos bajo una organización característica en este marco teórico; la transposición didáctica como lugar de producción de sentido. Los saberes basados en la experiencia, que hacen referencia a los principios de actuación profesional que mantiene el profesorado como resultado de su reflexión de la práctica misma y a los sentidos que dicha práctica provoca y forja a las categorías de enseñanza, las teorías implícitas que habitan en el campo cultural institucional y los guiones y rutinas que son construidos a partir de la historia de vida del profesorado. Perafán (en prensa) plantea además que una característica importante de este conocimiento es que "se refiere básicamente a la recuperación histórica y al desarrollo de lo que construye el profesorado en torno a las categorías que, de manera diferenciada, históricamente enseña" (p. 14). En la figura 1 se representa el conocimiento profesional específico. 


\section{El conocimiento profesional docente especifico asociado a categorías particulares}

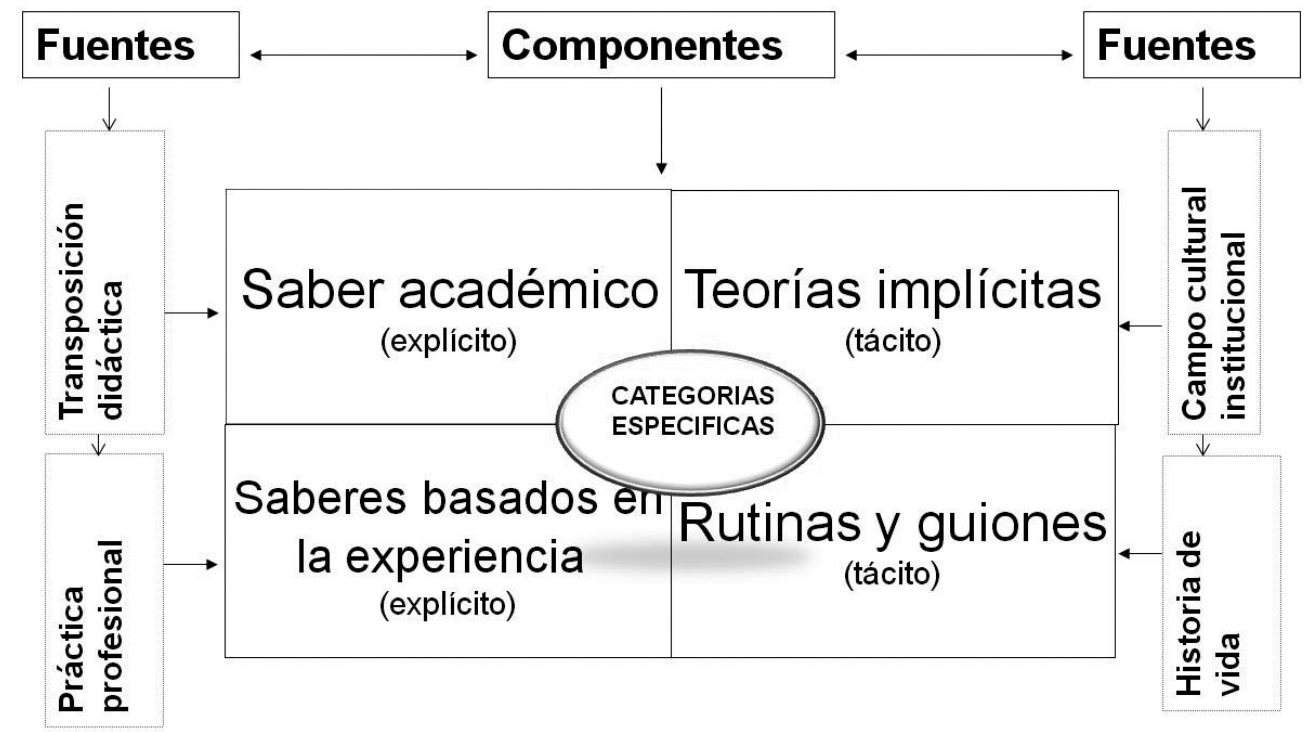

Figura 1. Fuente: "La transposición didáctica como estatuto epistemológico fundante de los saberes académicos del profesorado de ciencias" (Perafán, 2012).

En últimas, esta perspectiva ha mostrado, tanto desde un punto de vista conceptual (Perafán, 2004, 2013, 2015, en prensa), como con estudios de casos concretos (Ángel, 2013; Barinas, 2014; Barraza, 2014; Espinosa, 2013; Ortega, 2015; Perafán et al., 2015; Reina, 2014; Tinjacá, 2013), que se hace necesario realizar un giro interpretativo que reconozca, admita y reafirme históricamente, por una parte, la participación real y concreta del profesorado en la construcción del sentido de las categorías que enseña- lo cual significa reconocer que es constructor de un saber disciplinar que integra su conocimiento profesional-; y, por otra parte, la inalienabilidad del conocimiento disciplinar que se enseña en la escuela, y más concretamente en las aulas, de los procesos de producción discursiva y, por ende, del sentido que construye el profesorado. Para esto la categoría a la que hacemos alusión en estos momentos parece presentarse como una herramienta fundamental.
La tesis doctoral de la cual este documento constituye un insumo teórico importante (El conocimiento profesional específico del profesor de tecnología informática, asociado al concepto de tecnología), muestra algunos novedosos aportes relacionados con la aplicación de este marco teórico, desde el cual se intenta identificar, caracterizar e interpretar de manera específica el sentido que los profesores de tecnología e informática atribuyen al concepto de tecnología, desde sus prácticas de enseñanza, evidenciado con ello elementos que permiten distinguir el carácter disciplinar propio que mantiene el profesorado en su conocimiento profesional. Algunos de los avances que se desprenden de este estudio tienen que ver con la emergencia del sentido que los profesores atribuyen; ello legitima su aporte en términos de construcción de conocimiento de las áreas que enseñan, en este caso sobre el concepto de tecnología en el área de tecnología e informática. Algunos aspectos característicos propios de la condición integradora del concepto de tecnología empleado por el profesor para enseñar son: 


\section{La unidad representativa de sentido del aula de} clases. El concepto de tecnología como un cuerpo de conocimiento integrado se caracteriza por la naturaleza diversa y compleja que posibilita la emergencia situacional del sentido dentro del evento clase. La clase desde esta perspectiva es un acto de disposición y preparación colectiva para la determinación del saber. Por ello, la organización de este conocimiento debe reconocerse en el marco de una variedad de actos y determinaciones intencionadas que los sujetos partícipes de la clase aportan con el pleno de su subjetividad, para que de ello devengan actos como el de la enseñanza y del aprendizaje en función del concepto de tecnología, de tal forma que el conjunto de acciones explícitas o tácitas que hacen posible el evento denominado clase posibiliten también la construcción de este conocimiento. El concepto de tecnología de esta manera es producto del complejo mundo de relaciones que se entretejen en el evento clase, es decir, del aula de clase como unidad representativa que promueve constantemente la construcción de sentido.

2. La incidencia de las fuentes de saber que nutren el conocimiento del profesor y sus respectivos lugares de producción o estatutos epistemológicos fundantes (cf. Perafán, 2015, en prensa). Se asume que el concepto de tecnología que enseña el profesor es producto de la relación histórica que este ha mantenido con el saber sobre tecnología que enseña, y dicho saber se nutre y configura a partir de cada una de las fuentes de conocimiento constitutivas del saber del profesor. Hablamos de saberes académicos, saberes basados en la experiencia, teorías implícitas y guiones y rutinas, así como también de los lugares de producción de sentido o estatutos epistemológicos fundantes que posibilitan la construcción de la categoría tecnología. En este orden encontramos:

- La transposición didáctica que a nuestro juicio representa un lugar de producción de sentido escolar en el cual el profesor de tecnología e informática se realiza como sujeto de enseñanza que intencionalmente enseña un concepto de tecnología que educa sujetos. Esta característica que define aspectos de su subjetividad profesoral, anclados a una relación histórica de sentido con el objeto de saber denominado concepto de tecnología, advierte su separación y marca un grado importante de distinción con la categoría de tecnología producto del campo disciplinar que también se preocupa de su estructuración, mas no de la naturaleza epistemológica que envuelve su condición de categoría a ser enseñada; es decir, del nacimiento del concepto de tecnología bajo la aurora escolar y la intencionalidad de enseñanza que la define.

- La práctica profesional que trasciende de un escenario de actuación a toda una estructura generatriz de sentido sobre el hacer y la experiencia docente. En este caso también aporta elementos constitutivos de la subjetividad profesoral, validando a su vez la construcción de un concepto de tecnología producto de la experticia y experiencia que origina la práctica misma como escenario de razón.

- El campo cultural institucional como una red de relaciones de sentidos, que implícitamente aportan a la construcción del concepto de tecnología que se enseña en el aula. Esta red de sentidos implícitos recupera una serie de referentes teóricos deconstruidos a manera de tejido institucional que forja la comprensión y enseñanza de una categoría de tecnología propia de este escenario.

- Y la historia de vida como sustrato intelectual del cual este profesorado recupera su historia como sujeto perteneciente a una comunidad y a una cultura. Así se definen esquemas de actuación propios tanto de manera tácita como reprimida, que luego aparecen para enseñar la tecnología en la escuela.

De tal forma, el concepto de tecnología como saber propio del profesor es producto de todo un entramado de relaciones, de fuentes de saber y lugares de producción de sentido, que se organiza en el pensamiento del profesor para enseñar este concepto específico. 
3. Fenómeno discursivo de producción escolar de sentido (cf. Perafán, 2015, en prensa). Este tercer aspecto, que se articula con los dos anteriores, también explica el principio integrador del concepto de tecnología y obedece a una relación dialógica y dinámica propia de un diálogo deconstructivo de los cuatro tipos de saber visibles en la unidad de aula de clase en el dispositivo discursivo del profesor. Esto provoca la emergencia de una variedad de figuras literarias que proyectan las creaciones del profesorado para enseñar qué es tecnología en la escuela; hablamos de metáforas, símiles, imágenes, analogías, ejemplos, relatos, etc., elementos que identifican, guían y viabilizan la enseñanza de este contenido disciplinar escolar.

\section{Conclusiones}

Existe una comprensión naturalizada sobre la procedencia del carácter disciplinar del conocimiento del profesor, que lo determina marcadamente en los campos disciplinares propios de las disciplinas científicas, o en su defecto, bajo el dominio del saber producido por comunidades de expertos encargados del estudio de la enseñanza de las disciplinas científicas. Tal condición ha favorecido desde el ámbito profesional un tipo de reducción sobre las posibilidades de desentrañar y fijar la atención en la red de sentidos atribuidos por los profesores cuando enseñan las categorías de enseñanza propias de las disciplinas que circulan en el contexto escolar.

A pesar de los avances y el amplio despliegue que ha mantenido el programa internacional sobre el conocimiento del profesor en relación con las características epistemológicas que lo distinguen, existe una lectura restringida sobre el saber que produce el maestro como conocimiento propio que educa. Tales posicionamientos no resuelven por completo el problema de comprender la producción de conocimiento por parte del profesorado más allá del cómo en el sentido didáctico. El trabajo docente $y$ en especial con los objetos de saber se instala en una lógica operacional distinta de creación de sentido escolar y, por ende, bajo una intención de producción y enseñanza del saber diferente. Por ello, es necesario su compresión y estudio como un trabajo intelectual de generación de sentido propio, de carácter histórico y sistemático, que incide en la constitución de un corpus disciplinar particular que también enriquece los contenidos de enseñanza de las disciplinas escolares.

La apuesta investigativa en este orden debe jugarse por el reconocimiento y estudio del saber enseñado en medio de la relación histórica que ha mantenido el profesor con el conocimiento con que educa, y que entendemos se juega en medio de un cúmulo de atribuciones de sentidos propios producidos por los profesores que provocan la emergencia de sujetos aprendices de los contenidos de las disciplinas escolares.

De igual forma, las disciplinas escolares constituyen lugares académicos que declaran su separación y distinción especialmente por el lugar cultural del anclaje donde nacen y se reproducen (la escuela), comprendiendo también el hecho de que el profesorado responsable de su enseñanza representa un agente activo y visible en el enriquecimiento del conocimiento que funda determinada disciplina escolar, más allá de su participación en procesos relacionados con el cómo enseñar los contenidos constitutivos de las mismas.

En este orden, entendemos que los contenidos escolares representan una creación histórica de la propia escuela, por y para la escuela (Chervel, 1991), en el que se recupera el sentido que atribuye el profesor, como saber que educa y contribuye a la formación de sujetos. Este posicionamiento marca diferencias respecto de algunas posturas tradicionales que reconocen el saber proveniente del ámbito escolar -y en especial el que enseña el profesor-como un saber "banalizado", "vulgarizado" y además "deformado" de las disciplinas científicas, ya que su intención generatriz no precisa en ampliar la aurora de conocimiento disciplinar, sino que precisamente por ser de carácter escolar se ubica en el plano de los saberes que pretenden formar individuos bajo una disciplina escolar específica, asociada a un contenido escolar determinado, el cual ha sido construido en una comunidad de profesores. 
La categoría conocimiento profesional específico asociado a categorías particulares (Perafán, 2015, en prensa) se postula como cuerpo teórico adecuado que permite revalidar la relación histórica que ha mantenido el profesor con el conocimiento con que educa. Se evidencia que existen sentidos propios producidos por los profesores, que provocan la emergencia de sujetos aprendices de tales nociones escolares de enseñanza. Así por ejemplo, como se evidencia en el trabajo de investigación El conocimiento profesional específico del profesorado de tecnología e informática asociado al concepto de tecnología escolar, tal contenido de enseñanza desde su principio integrador se caracteriza por: a) el aula de clase como unidad representativa que promueve constantemente la construcción de sentido; b) la incidencia de los tipos de saber con sus respectivos estatutos epistemológicos fundantes (cf. Perafán, 2015, en prensa); c) un fenómeno discursivo de producción escolar de sentido (cf. Perafán, 2015, en prensa), que explica y articula con los otros dos componentes las creaciones del profesorado para enseñar el concepto de tecnología en la escuela. Hablamos de metáforas, símiles, imágenes, analogías, ejemplos, etc., figuras literarias que otorgan identidad epistemológica, guían y posibilitan la enseñanza de este concepto escolar.

Finalmente, entendemos que el conocimiento profesional del profesor debe comprenderse bajo procesos de interpelación subjetiva en el orden cultural, impregnados por la intencionalidad de la enseñanza que hace posible la emergencia de un conocimiento propio para la escuela, hecho que instala una apertura en la comprensión del carácter disciplinar de este conocimiento profesional.

\section{Referencias bibliográficas}

Ángel, Z. (2013). El conocimiento profesional específico del profesor de matemáticas asociado a la noción de número entero (tesis de maestría). Universidad Pedagógica Nacional, Bogotá, Colombia.

Barinas, V. (2014). El conocimiento profesional especifico del profesor de biología asociado a la noción de célula (tesis de maestría). Universidad Pedagógica Nacional, Bogotá, Colombia.
Barraza, V. (2014). El conocimiento profesional específico del profesorado de matemáticas asociado a la noción de función (tesis de maestría). Universidad Pedagógica Nacional, Bogotá, Colombia.

Bolívar, A. (2005). Conocimiento didáctico del contenido y didácticas específicas. Profesorado: Revista de Currículum y Formación del Profesorado, 2(9), 1-39. Recuperado de http://www.ugr.es/ recfpro/ rev92ART6.pdf.

Bolívar, A. (2008). Didáctica y currículum: de la modernidad a la postmodernidad. Málaga: Aljibe.

Camilloni, A. (Comp.) (2007). El saber didáctico. Argentina: Paidós.

Carr, W. y Kemmis, S. (1988). Teoría crítica de la enseñanza. La investigación en la acción en la formación del profesorado. Barcelona: Martínez Roca.

Chervel, A. (1991). Historia de las disciplinas escolares. Reflexiones sobre un campo de investigación, Revista de Educación, 295, pp. 7-37 y 296, pp. 59-111.

Chevallard, Y. (1991). La transposición didáctica. Del saber sabio al saber enseñado. Buenos Aires: Aiqué.

Connelly, F. M. y Clandinin, D. J. (1984). Personal practical knowledge at Bay Street School: ritual, personal philosophy and image. En R. Halkes, y J. K. Olson, (eds.), Teacher thinking. A new perspective on persisting problems in education (pp. 134-148). Lisse: Sweets and Zeitlinger.

Cuesta, R. (1997). Sociogénesis de una disciplina escolar: la hhistoria. Barcelona: Pomares-Corredor.

Cuesta, R. (2014). Genealogía y cambio conceptual. Educación, historia y memoria. Archivos Analíticos de Políticas Educativas, 22(23). doi:http://dx.doi. org/10.14507/epaa.v22n23.2014.

Dolz, J. (2011). Describir la actividad docente: un punto de vista didáctico para comprender el trabajo del profesor en el aula (pp. 97-110) En J. Villanueva, D. Álvarez y R. Rickenmann (eds.) L'activitat docent. Intervenció, innovació, investigación. Girona: Documenta Universitaria.

Elbaz, F. (1981). Teacher's "practical of knowledge": a Case. Curriculum Inquiry, 11(1), 43-71.

Espinosa, S. (2013). El conocimiento profesional especifico de los profesores de preescolar y primaria asociados a la noción de escritura (tesis de maestría). Universidad Pedagógica Nacional, Bogotá, Colombia. 
Furió, C. (1994). Tendencias actuales en la formación del profesorado de ciencias. Enseñanza de las Ciencias, 2(2), 188-199.

García, M. (1997). Conocimiento profesional del profesor de matemáticas. El concepto de función como objeto de enseñanza-aprendizaje. Sevilla: Universidad de Sevilla.

Gil-Pérez, D. (1994). Relaciones entre conocimiento escolar y conocimiento científico. Investigación en la Escuela, 23, 17-32.

Gimeno, J. y Pérez, A. I. (1988). Pensamiento y acción en el profesor: de los estudios sobre la planificación al pensamiento práctico. Infancia y Aprendizaje, 42, 37-63.

Giroux, H. A. (1997). Los profesores como intelectuales. (1. ${ }^{\mathrm{a}}$ reimp.). Barcelona: Paidós.

Gómez, M. y Alzate, M. (2010). Saberes, disciplinas y disciplinas escolares: diferentes sentidos para las didácticas. Cultura del Cuidado Enfermería, 7(1), 37-52.

Goodson, I. F. (1991). La construcción social del currículum. Posibilidades y ámbitos de investigación de la historia del currículum. Revista de Educación, 295, 7-37.

Goodson, I. (1995): Historia del Currículum. La construcción social de las disciplinas escolares. Barcelona: Pomares-Corredor.

Jackson, Ph. W. (1968). La vida en las aulas. Madrid: Morata.

Julia, D. (2000). La construcción de las disciplinas escolares en Europa. En J. Ruiz Berrio (ed.), La cultura escolar en Europa. Tendencias históricas emergentes. Madrid: Biblioteca Nueva.

Marcelo, G. C. (1993). Cómo conocen los profesores la materia que enseñan. Algunas contribuciones de la investigación sobre conocimiento didáctico del contenido. En: Montero, L. y Vez, J. (Eds.). Las didácticas específicas en la formación del profesorado. Santiago de Compostela: Tórculo. pp. 151-185.

Marcelo, G. C. (2002). La investigación sobre el conocimiento de los profesores y el proceso de aprender a enseñar. Una revisión personal. En G. A. Perafán y A. Aduriz-Bravo (eds.), Pensamiento y conocimiento de los profesores. Debate y perspectivas internacionales. Bogotá: UPN-Colciencias.
Martínez, C. (2000). Las propuestas curriculares sobre el conocimiento escolar en el área de conocimiento del medio: dos estudios de caso en profesores de primaria (tesis doctoral). Universidad de Sevilla. España.

Martínez, C. (2005). De los contenidos al conocimiento escolar en las clases de ciencias. Educación y Pedagogía, 17(43), 151-161.

Ortega, J. (2015). El conocimiento profesional específico del profesorado de tecnología e informática asociado al concepto de tecnología escolar [Informe de tesis de grado del Doctorado Interinstitucional en Educación]. Manuscrito inédito. Universidad Pedagógica Nacional de Colombia, Bogotá, Colombia.

Perafán, G. A. (2004). La epistemología del profesor sorbe su propio conocimiento profesional. Bogotá: Universidad Pedagógica Nacional.

Perafán G. A. (en prensa). El conocimiento profesional docente: nuevas perspectivas epistemológicas y metodológicas. Bogotá: UPN. Material de trabajo del Seminario Doctoral DIE.

Perafán, G. A. (2012). La transposición didáctica como estatuto epistemológico fundante de los saberes académicos del profesorado de ciencias (III). Ponencia presentada en la II Conferencia Latinoamericana del International History, Philosophy, and Science Teaching Group. Octubre 3-6. Mendoza, Argentina.

Perafán, G. A. (2013). La transposición didáctica como estatuto epistemológico fundante de los saberes académicos del profesor. Folios, 37, 83-93.

Perafán, G. A. (2015). Conocimiento profesional docente y prácticas pedagógicas. El profesorado como productor de conocimiento disciplinar-profesional. Bogotá: Aula de Humanidades.

Perafán, G. A.; Sánchez, D. A.; Castilllo, P.; Barinas, V.; Reina, Y. y Neusa, D. C. (2015). El conocimiento profesional específico del profesorado de biología asociado a la noción escolar de célula: estudio de caso múltiple. Universidad Pedagógica Nacional de Colombia-cIUP. Informe final de investigación.

Perafán G. A. y Tinjacá, F. M. (2014). Aspectos generales y primeros avances para el encuadre de una investigación sobre el conocimiento profesional específico del profesorado de química asociado a la noción de nomenclatura química. Educación 23, 44, 48-64. 
Ponte, J. (2012). Estudiando el conocimiento y el desarrollo profesional del profesorado de matemáticas. En N. Planas (comp.), Teoría, crítica y práctica de la educación matemática. Barcelona: Grao.

Popkewitz, T. (2004). The alchemy of the mathematics curriculum: Inscriptions and the fabrication of the child. American Educational Research Journal, 41(4), 3-34.

Popkewitz, T. S. (2010). Estudios curriculares y la historia del presente. Profesorado. Revista de Currículo y Formación del Profesorado, 14(1), 355-370.

Porlán, A. R. y Rivero, G. A. (1998). El conocimiento de los profesores. Revista de Educación, 295, 7-37.

Schön, D. A. (1998) El profesional reflexivo. Cómo piensan los profesionales cuando actúan. Barcelona: Paidós.

Reina, M. (2014). El conocimiento profesional específico del profesorado de tecnología asociado a la noción de diseño (tesis de maestría). Universidad Pedagógica Nacional, Bogotá, Colombia.

Shulman, L. (1986). Those who understand: Knowledge growth in teaching. Educational Researcher, 15(2), 4-14.
Shulman, L. S. (1987). Knowledge and teaching: foundations of new reform. Harvard Educational Review, 57(1), 1-22. [Edición en español: (2005). Conocimiento y enseñanza: fundamentos de la nueva reforma. Profesorado. Revista de Currículum y Formación del Profesorado, Granada, 9 (2)].

Tanner D. y Tanner L. (1990). History of the school curriculum. Nueva York-Londres: MacMillan/Collier Macmillan.

Tinjaca, F. (2013). El conocimiento profesional específico del profesorado de química asociado a la noción de nomenclatura química (tesis de maestría). Universidad Pedagógica Nacional, Bogotá, Colombia.

Viñao, A. (2012). Historia de las disciplinas, profesionalización docente y formación de profesores: el caso español. Pro-Posições, 23, 3(69), 103-118.

Wilson, S. M.; Shulman, L. S. y Richert, A. E. (1987). "150 different ways" of knowing: Representations of knowledge in teaching. En J. Calderhead (ed.), Exploring Teacher Thinking (pp.104-124). Londres: Cassell. 